\title{
Défis éthiques et risques pratiques du terrain en situation de développement ou d'urgence humanitaire
}

Introduction

Sylvie Ayimpam, Magali Chelpi-den Hamer et Jacky Bouju

\section{OpenEdition}

Journals

Édition électronique

URL : http://journals.openedition.org/anthropodev/292

DOI : 10.4000/anthropodev.292

ISSN : 2553-1719

Éditeur

APAD - Association pour l'anthropologie du changement social et du développement

Édition imprimée

Date de publication : 1 septembre 2014

Pagination : 21-41

ISBN : 9791093476018

ISSN : 2276-2019

Référence électronique

Sylvie Ayimpam, Magali Chelpi-den Hamer et Jacky Bouju, « Défis éthiques et risques pratiques du terrain en situation de développement ou d'urgence humanitaire », Anthropologie \& développement [En ligne], 40-41 | 2014, mis en ligne le 01 septembre 2016, consulté le 20 avril 2019. URL : http:// journals.openedition.org/anthropodev/292 ; DOI : 10.4000/anthropodev.292

La revue Anthropologie \& développement est mise à disposition selon les termes de la Licence Creative Commons Attribution 4.0 International. 


\title{
Les terrains du développement et de l'humanitaire : \\ convergences et renouveau réflexif
}

\author{
Introduction : \\ Défis éthiques et risques pratiques du terrain \\ en situation de développement ou d'urgence humanitaire
}

Sylvie Ayimpam, Magali Chelpi-den Hamer, Jacky Bouju

De l'image, encore très ancrée, de l'anthropologue installé dans un village pendant des années pour étudier en profondeur une culture exotique, on est passé à une anthropologie plus "rapide", portée sur des enjeux de société actuels, médiatiques parfois, et sur des terrains tant proches que lointains. Ce changement est important à noter parce qu'il souligne de nouveaux enjeux pour la discipline, notamment celui de savoir s'articuler avec les études sociologiques qualitatives et la géographie sociale quand elles s'inspirent des mêmes terrains. On s'accorde aujourd'hui à considérer que, sous l'effet de la mondialisation et des conjonctures nouvelles auxquelles sont confrontées les populations, les terrains se sont " métamorphosés $»^{1}$ (Copans et Genest, $2000: 5$ ). C'est ainsi que depuis une quinzaine d'années, les nouvelles générations d'anthropologues ont été particulièrement attentives à la multiplicité des discours, à la diversité des intérêts locaux, à la complexité du "réel des Autres " $^{2}$. Elles se sont révélées également très réflexives sur le thème de la rencontre ethnographique et des difficultés que pose la communication interculturelle, et très attentives aussi à décrire l'enquête en n'oubliant pas la participation des assistants

\footnotetext{
${ }^{1}$ Le lecteur intéressé trouvera un historique complet de la notion de terrain (fieldwork) et de son évolution ainsi qu'une analyse critique des définitions successives qu'en a donné l'anthropologie dans Robben et Sluka (2007), "Fieldwork in Cultural Anthropology : An Introduction » : 6-22. Pour une discussion critique de la notion de terrain en anthropologie voir Pulman (1988), Albera (2001), Boumaza et Campana (2007), Robben et Sluka (2007).

${ }^{2}$ Un clin d'œil à Jean-Pierre Olivier de Sardan!
} 
de recherche et des informateurs dans le travail de production et de restitution de l'information (Robben et Sluka, 2007).

L'anthropologie du développement a participé à ce renouveau en explorant de nouvelles manières de faire de l'ethnographie dans des situations d'interventions courtes et planifiées opérant dans des contextes parfois difficiles. C'est ainsi qu'aujourd'hui, nombre d'anthropologues sont engagés dans des formes de recherche collaboratives et participatives dans lesquelles l'information est un processus de co-production épistémologique issue d'une interaction dialogique répétée (Pink, 2000, 2006). Mais, dans la dernière décade, l'accroissement des foyers de tensions s'est accompagné localement de guerres civiles avec leurs lots d'atrocités collectives, de génocides, et de violations récurrentes des droits de l'Homme dans le monde, qui ont transformé quantités d'êtres humains en réfugiés ou qui les ont contraints à la migration forcée ou à l'exode rural. L'exposition croissante des situations d'enquête sur le terrain à de multiples risques a incité un nombre croissant de chercheurs à prendre conscience des dimensions éthiques, morales et politiques de l'enquête. Cette prise de conscience les a conduits à étudier, entre autres, la guerre, les catastrophes, la violence, la souffrance sociale et les traumatismes culturels, la résilience et la reconstruction sociale ${ }^{3}$.

La multiplication des situations "d'urgence", de "conflit" ou de " post-conflit " a soumis l'enquête de terrain en situation d'urgence humanitaire ou de développement à de nouveaux risques auxquels elle a dû s'adapter. Les politiques de l'humanitaire s'étant rapprochées des politiques de développement, l'anthropologie de l'action humanitaire s'est rapidement réapproprié nombre d'idées issues des travaux en anthropologie du développement (les questions de capture et de manipulation de l'intervention "par le haut " et "par le bas", la question du degré d'enchâssement dans l'environnement immédiat, les jeux d'acteurs et les jeux de pouvoir). Elle participe pleinement aujourd'hui aux enjeux de positionnement de la discipline, notamment pour ce qui concerne la mise en débat des résultats de recherche sur des objets d'enquête en général for-

\footnotetext{
${ }^{3}$ On est bien loin ici de la conception postmoderne du terrain qui n'aurait d'autre réalité que celle d'une "fiction " reconstruite par un travail d'écriture et de distanciation partielle et partiale.
} 
tement médiatisés. De nouveaux objets d'étude émergent donc et prennent la forme d'un retour réflexif sur des expériences personnelles d'implication au sein des institutions d'aide. L'étude des enjeux et des logiques internes à ces institutions fait aussi partie de cette nouvelle objectivation, tout comme les réflexions plus épistémologiques qui touchent à l'étude de la production d'une myriade de savoirs-experts qui dérivent des interventions humanitaires et de développement.

Dans ces nouveaux champs qui sont aujourd'hui explorés par les anthropologues du développement et de l'humanitaire, on mesure mieux encore à quel point le chercheur est en interaction permanente avec une gamme élargie d'acteurs également investis dans le champ de l'aide, et à quel point il doit négocier sa place et ses relations, voire ses objets de recherche. À des degrés variés donc, la production anthropologique sur ces nouveaux terrains se retrouve modelée par ces interactions, voire en partie coproduite par cet ensemble d'acteurs opérant et/ou enquêtant sur les mêmes terrains. On est là donc pleinement dans un champ "perturbé " par la situation d'intervention et où cette perturbation a finalement tendance à devenir la norme puisque de nos jours, dans les contextes de crise, les champs vierges de toute intervention d'aide sont rares. Tous ces acteurs observent et enquêtent sur les mêmes terrains, mais avec des objectifs, des moyens et des méthodes différents, ce qui n'est pas sans entrainer une certaine confusion chez les sujets enquêtés. Cela peut aussi amener à une certaine ambiguïté d'autant plus grande quand le chercheur endosse simultanément le rôle $\mathrm{d}^{\prime}$ expert ${ }^{4}$.

Pourtant, force est de constater que, mis à part l'ouvrage de référence de Florence Bouillon, Marion Frésia et Virginie Tallio (2005), le monde francophone du développement et de l'humanitaire est resté peu touché par la vague réflexive anglo-saxonne. Bouillon, Frésia et Tallio abordent la question des « terrains sensibles " par trois entrées : certains terrains sont

${ }^{4}$ Les réflexions actuelles sur les enjeux liés à la double posture chercheur/expert, par exemple, en sont une bonne illustration. Mais cette double posture obéit aussi à une stratégie sécuritaire qui impose sa nécessité quand il existe des possibilités de transports onusiens permettant d'accéder à des zones où il serait dangereux d'aller par ses propres moyens. Cette question sera traitée minutieusement dans un ouvrage collectif en préparation sous la coordination de Philippe Lavigne Delville et de Marion Frésia. 
qualifiés de sensibles au sens où les objets d'étude sont caractérisés par des pratiques illégales ou informelles et où les principaux sujets d'étude sont souvent marqués par une forte stigmatisation sociale (squatteurs, migrants en transit, sans-papiers, etc.); d'autres terrains (parfois les mêmes) sont dits "sensibles " au sens où, méthodologiquement, il s'avère difficile pour le chercheur de mettre en œuvre une observation prolongée dans le temps (à cause de la situation de clandestinité, à cause de lieux d'enquêtes et d'observations sujets à des fermetures soudaines, à cause des relations de confiance rendues plus difficiles par des mises en danger immédiates sur les sujets d'étude, ou tout simplement à cause d'un régime autoritaire qui freine la liberté d'expression) ; d'autres terrains, enfin, sont qualifiés de "sensibles " parce qu'ils expriment de manière très concrète la fin du monopole du chercheur sur certains sujets. En effet, de plus en plus, dans des lieux d'enquêtes marginaux, conflictuels ou à forts enjeux sociaux - comme les zones de conflits, de non droit, soumises à un régime autoritaire, les camps de réfugiés, les situations d'urgence humanitaire, avec tout le tapage médiatique qui les accompagnent - les chercheurs doivent composer avec d'autres acteurs médiateurs d'information (ONG locales, agences internationales, travailleurs sociaux, journalistes, etc.). La transversalité complexe et multidimensionnelle des terrains a des implications méthodologiques nouvelles qui obligent le chercheur et l'informateur à s'impliquer chacun dans la vie de l'autre, de telle sorte qu'ils partagent un espace de subjectivité dans lequel ils co-construisent le savoir ethnographique. Mais, la patiente construction d'un espace d'intersubjectivité entre le chercheur et les participants à la recherche est bien souvent « minée " (Albera, $2001: 5)$. Moins, cependant, par la posture et la méthode de l'anthropologue, que par la métamorphose des conditions de la confrontation interculturelle qui se révèle en situation d'enquête (Pulman, 1988 : 28). Certains chercheurs doutant de leur capacité méthodologique et éthique à protéger leurs informateurs sans se mettre eux-mêmes en danger estiment qu'il n'est pas possible d'enquêter sur certains terrains. Si leur prudence est louable, il reste nécessaire de s'interroger sur le 'risque'. Qu'entend-on par là et comment en faire une analyse pertinente?

En organisant en 2013 un colloque international sur le thème " Enquêter en contexte de développement ou d'urgence ", I'APAD avait, entre autres ambitions, de faire un état de la situation dans le monde francophone. C'est ainsi que les chercheurs et les praticiens invités à participer 
au colloque ont été conviés à porter un regard critique réflexif sur leurs expériences et leurs pratiques récentes du terrain. Les communications, passionnantes, ont été révélatrices de manières de faire et de bricolages, mais aussi de doutes et de questions sans réponse, qui confirmaient amplement ce qu'avaient déjà montré les travaux anglophones.

\section{Le risque du terrain et sa perception}

Une première sorte de difficulté associée à la situation d'enquête sur le terrain tient à l'expérience de la tension interculturelle (psychologiquement risquée) que le chercheur doit résoudre en gérant au mieux les rapports de distance et de proximité dans ses interactions quotidiennes. Mais, cette expérience empirique a le mérite de lui révéler les stratégies d'évitement et de surveillance employées par les uns et les autres, et les conséquences de l'enchaînement des attributions d'intentions favorables ou malveillantes sur la place qu'il est censé occuper. L'expérience du terrain se joue alors dans l'ensemble des relations d'enquête qu'il choisira de maintenir (au prix de subir les tensions liées à la suspicion permanente d'être considéré comme un agent, un journaliste, un "indic " ou un " espion " commandité par le projet, la hiérarchie, l'administration ou le bailleur de fonds et d'être traité en conséquence) ou de réduire (au prix de l'encliquage, du parti-pris ou de la prise de risques physiques ou juridiques au cas où il serait considéré par les autorités locales comme un agitateur ou un complice d'activités illégitimes voire illégales).

La perception des risques liés à un terrain dépend dès lors de la compréhension fine de ce qui est en jeu dans l'expérience que vit le chercheur dans la situation d'enquête. Elle dépend donc directement de son implication de chercheur dans son objet de recherche, des rôles qu'il a déjà tenu auparavant dans ce milieu local, des identités imposées qu'il a dû négocier et des rôles nouveaux qu'il se sent prêt à assumer. Cette sorte de difficulté est subjective et systématique, aussi personnelle qu'inévitable. Daniel Bizeul (2007: 73) rappelle à ce propos que le degré de difficulté rencontré par le chercheur ne peut être détaché de ce qu'il est, de sa trajectoire initiale, de ses expériences de vie, du style de recherche pratiqué dans son laboratoire, de son identité psychologique et morale aux yeux des autres. La situation d'enquête sur le terrain est toujours une expérience person- 
nelle forte, "à la fois l'évidence d'une rupture et l'incertitude quant à sa signification »(Bensa et Fassin, 2002 : 17). Ce n'est qu'a posteriori que l'anthropologue peut en reconstruire la signification et en comprendre les vraies difficultés.

Les questions de confidentialité, de respect de l'anonymat et de neutralité axiologique se posent de manière nouvelle dans ces nouveaux champs et plusieurs difficultés pratiques ressortent des contributions. Changer les noms et les lieux ne suffit pas à rendre les informateurs anonymes et un chercheur consciencieux ne devrait pas se contenter d'un anonymat "de façade " (Weber, 2008). Pour autant, il faut bien y réfléchir car en gommant nuances et liaisons, l'anonymisation peut conduire à un appauvrissement de l'analyse.

\section{Terrains à risques et objets de recherche sensibles}

La question de la perception subjective du risque du terrain ayant été posée, il est alors possible d'envisager ce qui peut constituer un risque objectif sur le terrain et de considérer les enjeux méthodologiques et éthiques et les dilemmes qu'ils posent à l'action d'enquêter. Nous n'aborderons pas, dans ce numéro, les défis éthiques et méthodologiques particuliers que pose l'enquête sur le terrain en contexte de guerre ou de violence physique avérée $e^{5}$. Les textes proposés ici analysent plutôt des situations d'enquête en situations de risques "modérés" tant pour le chercheur que pour les participants à la recherche. En s'engageant dans ce genre de situations d'enquête, le chercheur accepte d'emblée de se trouver dans des espaces sociaux embarrassants dont certains sont plus difficiles à occuper que d'autres.

Ces risques tiennent à la nature de l'objet de recherche qui peut être politiquement "sensible ", socialement "illégitime " ou religieusement " tabou ». La difficulté particulière de ces objets de recherche est d'être caractérisée par des luttes conceptuelles qui les rendent hostiles, fuyants, " saturés " d'enjeux sémantiques qui mettent à mal les canons de la mé-

${ }^{5}$ Cette situation est bien analysée notamment dans les travaux de Nordstrom et Robben (1995), Agier (1997), Le Palec et Luxereau (1999), Nilan (2002), Sriram et al. (2009). 
thode (neutralité du chercheur, rigueur méthodologique, etc.), comme on le verra avec les contributions de Ramatou Ouedraogo et de Hadiza Moussa, dans lesquelles les chercheures se trouvent prises dans des luttes de significations très tendues, à propos de l'avortement dans un contexte de stigmatisation communautaire ou encore à propos de l'excision dans un contexte de religiosité exacerbée. Ces risques peuvent aussi tenir aux contextes autoritaires dans lesquels se déroule l'enquête comme c'est le cas avec les contributions de Molly Sundberg et de Mikaëla Le Meur, ou de guerre civile rendant l'accès au terrain impossible, qu'illustre si bien I'analyse de Sten Hagberg et de Gabriella Körling.

Mais, ces risques tiennent aussi aux populations et aux catégories sociales méprisées, abandonnées ou stigmatisées qui sont concernées par l'enquête. Ainsi, une situation locale d'extrême tension politique ou d'exacerbation des haines religieuses, susceptible de basculer à tout instant dans l'expression d'une violence brutale, constitue à l'évidence un risque objectif tant pour le chercheur que pour les sujets enquêtés. Ce type de situation engendre des émotions personnelles qu'il faut gérer et une adaptation de l'enquête qui peut prendre toutes les formes imaginables jusqu'à sa suspension pure et simple. Quand le chercheur est informé par avance de l'atmosphère empoisonnée qui règne sur "le terrain ", il peut malgré tout choisir d'y aller quand même. Mais, en s'engageant dans ce genre de situations d'enquête, il accepte d'emblée de se trouver dans des espaces sociaux embarrassants dont certains sont plus difficiles à occuper que d'autres à cause des risques qu'ils représentent ${ }^{6}$. En témoigne l'expérience de Sylvain Batianga en République centrafricaine qui s'est trouvé pris dans une situation d'enquête " minée " par la contrainte politique et la suspicion contre "les » humanitaires. Plusieurs contributions font allusion à la difficulté de gérer l'empathie et les émotions qui découlent des interactions sur le terrain d'enquête. Non seulement faut-il que les acteurs consentent à être utilisés comme sujets

${ }^{6}$ Un défi majeur posé à la réflexivité du chercheur travaillant sur un terrain "à risque » est d'éviter deux écueils : le premier consisterait à tomber dans un discours narcissique faisant de lui une sorte de surhomme bravant des risques pour le seul bien de diffuser un savoir difficilement appréhendable ; le second serait de tomber dans une introspection excessivement sur-réflexive qui n'apporterait pas grand-chose à la solution des questions méthodologiques et éthiques en jeu. 
d'observation, mais il faut aussi de manière très précautionneuse anticiper les conséquences de ce consentement afin de les protéger des répercussions potentielles de leur implication. C'est le principe du "do no harm », qui est encore plus difficile à atteindre dans les zones de post-conflit à cause de contextes politiques fortement polarisés, de la présence de plusieurs forces armées et tout simplement de la difficulté générale de prévoir les événements à court et moyen terme. La gestion des risques, notamment ceux que le chercheur fait prendre à ses interlocuteurs et ceux qu'il prend lui-même, fait également débat.

Les contributions réunies dans ce volume constituent autant d'interrogations différentes des défis éthiques et des risques pratiques rencontrés sur le terrain qui peuvent être résumées de la manière suivante:

- Comment s'informe et se déforme l'approche méthodologique dans des contextes autoritaires où l'enquête n'est pas bienvenue et la prise de parole sur-contrôlée?

- Quelles réflexions méthodologiques et éthiques particulières se dégagent quand on enquête dans des lieux pouvant devenir dangereux, en situation de pré-conflit ou de post-conflit ?

- Peut-on vraiment enquêter sur tout objet de recherche quelle que soit sa sensibilité ?

- Enfin, jusqu'où peut-on aller sans enquête in situ ? Quels sont les enjeux méthodologiques et éthiques du suivi à distance pour la discipline?

Enquêter dans des contextes autoritaires où l'enquête n'est pas bienvenue et la prise de parole sur-contrôlée

Enquêter en contexte autoritaire est ainsi bien souvent un exercice complexe et délicat et plusieurs sujets d'étude peuvent clairement être considérés sensibles par les pouvoirs publics locaux comme en témoignent les deux contributions de Mikaëla Le Meur et de Molly Sundberg. Leurs contributions retracent les tribulations d'enquête en régime autoritaire et là où la prise de parole reste encore sur-contrôlée sur certains sujets (telle l'étude de projets gouvernementaux). Leur problème principal fut d'arriver à collecter des informations qui soient les moins manipulées possibles dans un contexte très défavorable. 
Explorant la question de la gestion des déchets en milieu semi-urbain, dans le cadre d'un programme de développement mené conjointement par une administration provinciale vietnamienne et un organisme de coopération européen, Mikaëla Le Meur, qui s'est trouvée confrontée à des difficultés pratiques d'accès à l'information, nous livre une belle analyse de ses hésitations méthodologiques. Dans le contexte vietnamien, c'est le gouvernement qui " décrète le réel ». L'approche anthropologique dans l'exploration des pratiques du quotidien, par la distanciation qui lui est propre, pourrait amener à remettre en question la politique du régime, et peut ainsi se révéler très problématique. Les notions de confiance, de réputation, de confidentialité revêtent ainsi une dimension particulière en terrain autoritaire, du fait des risques que le chercheur fait prendre à ses interlocuteurs et à lui-même. Le Meur mettra ainsi en avant l'aide opportune d'une logeuse qui, contrairement aux autres avant elle, ne craignait pas de se faire intimider par les policiers locaux, étant elle-même fonctionnaire communale avec son père représentant de quartier. Enquêter en tant que chercheur " infiltré » dans ce genre d'institution peut donc devenir assez délicat une fois que l'on aborde la question de la restitution qui, dans une certaine mesure et dans certaines cultures, peut véritablement être interprétée comme une menace pesant sur la cohésion institutionnelle.

Sa contribution permet de souligner deux éléments fondamentaux de la relation d'enquête : d'abord, la nécessité de s'interroger constamment sur la méthodologie en tant que telle en n'hésitant pas à réviser son approche initiale pour 'coller' au mieux aux sensibilités locales; ensuite, la nécessité de trouver des intermédiaires pertinents, capables d'ouvrir les bonnes portes. L'auteure précise que sa situation d'enquête n'a jamais pu être explicitée auprès des membres de l'institution; c'est seulement en partageant le quotidien des réunions internes, informelles et des activités officielles qu'elle cherche à rendre compte des enjeux de pouvoir liés à l'accès, à la circulation des contenus et à l'instrumentalisation des informations. Sur ce dernier point en particulier, la manipulation du chercheur par certains informateurs - que ce fût à des fins de contrôle du contenu des informations collectées, à des fins de promotion sociale individuelle ou à des fins plus sournoises, dans le but de faire endosser au chercheur des casquettes qui ne sont pas les siennes - est un élément qui ressort de plusieurs contributions. Ce problème d'instrumentalisation du chercheur 
n'est pas propre aux terrains à risques, mais sur ces terrains et ces thématiques particulières, les auteurs sont peut-être plus conscients d'être l'objet de stratégies manipulatoires. Une conséquence de cette situation est d'entrainer une complexification des jeux sociaux du don et du contredon. Ceci n'est pas sans rappeler la description par Rosalie Wax du jeu social qui caractérise l'interaction entre enquêteur et enquêté comme une partie de poker dont les règles seraient redéfinies à chaque nouvelle rencontre. A chaque nouvelle interaction, les deux acteurs s'entendent pour jouer ensemble, mais en négociant la co-construction des règles de leur échange de telle sorte que puissent s'imposer les règles que chacun considère comme non négociables (Wax, 1986 : 54).

Alors même que la collecte des déchets dysfonctionnait gravement dans le quartier, Mikaëla Le Meur décrit le "tabou» qui entourait l'évocation de ce sujet dans les conversations, à tel point que dans des lieux d'échanges informels comme les cafés ou les bars locaux, des personnes extérieures pouvaient intervenir dans la conversation en faisant remarquer à l'enquêté " qu'il parle trop ». L'interlocuteur du chercheur est ainsi constamment tiraillé entre ce qu'il souhaiterait partager et ce qu'il peut réellement exprimer sans s'attirer les foudres de l'appareil policier. La présence d'un " chaperon " officiel lors d'entretiens formels est d'ailleurs caractéristique de cette volonté d'encadrer le contenu de l'information officiellement donnée au chercheur. Pour autant, il ne faut pas oublier que l'un des rôles principaux de ces chaperons est de dédouaner l'enquêté de manière visible, en rendant impossible toute accusation ultérieure de conspiration contre le régime. L'enjeu principal donc, dans ces types de terrains où les interactions et les conversations sont très surveillées et réglementées, est d'arriver à créer un espace propice à l'émergence d'un discours moins stéréotypé. Les détours de langage, les plaisanteries, même anodines, apparaissent alors, dans un tel contexte de rareté du discours spontané, comme des éléments précieux que le chercheur est avide de collecter.

La contribution de Molly Sundberg rapportant une expérience de recherche sur un programme d'éducation civique au Rwanda - appelé Itorero - souligne, elle aussi, les défis d'une recherche entreprise dans un contexte social et politique caractérisé par la méfiance, la surveillance politique, la violence et le contrôle. Le programme d'éducation civique 
Itorero, qui aborde des sujets controversés voire contestés, tels que l'identité ethnique, les causes du génocide, les politiques et les lois sur la justice et la réconciliation, etc., suscite la méfiance et la suspicion du pouvoir. Sundberg indique que des rumeurs insistantes de surveillance policière (caméras de surveillance dans les restaurants, et micros implantés dans certains hôtels) circulaient parmi ses amis et ses informateurs rwandais, qui couraient le risque d'être convoqués par les services de renseignement et les services secrets du gouvernement à cause des délateurs existant dans leur communauté locale. Dans un tel contexte, donner un témoignage présente un risque pour les informateurs du chercheur qui développent alors des moyens de communications alternatifs. Elle a dû affronter en permanence l'autocensure ${ }^{7}$ de ses interlocuteurs qui se manifestait sous forme de silences, d'omissions, de dictons et plus particulièrement de rumeurs alarmantes. Dans ses interactions au quotidien avec les voisins, les autorités ou encore les participants au programme d'éducation civique Itorero, il n'était pas rare que l'on réponde à ses questions par une autre question, par un proverbe ésotérique ou de manière culturellement codée. Malgré l'aide de ses assistants de recherche pour les comprendre, les sens de ces réponses sont souvent restés énigmatiques. Le chercheur réalise alors que l'énoncé de tout témoignage sincère ne peut provenir que de la longue et patiente construction d'une relation de confiance qui passe par l'acceptation et l'interprétation des silences et des omissions, des rumeurs et des proverbes.

\section{Enquêter dans des lieux pouvant devenir dangereux, en situation de pré-conflit ou de post-conflit}

Dans le contexte socialement délétère d'une situation post-conflit en République centrafricaine, Sylvain Batianga témoigne d'une expérience sensiblement différente puisque l'Etat est absent. La situation d'enquête, " minée » par la suspicion et la contrainte politique, rapporte les réactions agressives de ses interlocuteurs alors qu'il revenait travailler comme cher-

\footnotetext{
${ }^{7}$ Molly Sundberg souligne que plusieurs chercheurs se sont plaints en travaillant au Rwanda de ne recevoir que des réponses " conformes aux attentes de l'Etat ", dès lors qu'ils abordaient avec leurs interlocuteurs des questions considérées comme politiquement sensibles.
} 
cheur après avoir régulièrement séjourné dans la zone comme collaborateur des organisations humanitaires. Découvrant avec stupéfaction la conception négative que la population locale se faisait du personnel humanitaire, il a décidé de changer l'objet de son investigation dans un souci d'apaisement, provoquant ce faisant un basculement réflexif de son analyse. Il est ainsi passé de l'analyse de la vulnérabilité des personnes déplacées et prises en charge par des organisations humanitaires, à l'analyse de sa propre vulnérabilité sur le terrain. En prenant de la distance avec l'émotion suscitée par l'agression verbale dont il a été l'objet, le chercheur a pu accéder à un univers de représentations locales de l'intervention humanitaire dont il ne soupçonnait pas a priori l'existence : à savoir que les organisations humanitaires entretiendraient l'insécurité afin de se reproduire par le renouvellement de leurs financements.

Le contexte ultra-militarisé, ultra-politisé, ultra-polarisé de terrains sous perfusion humanitaire après avoir été sérieusement troublés par des conflits violents influence fortement l'enquête et affecte la qualité des données collectées. L'approche narrative communément choisie invite les personnes à se remémorer les événements violents auxquels elles ont participé à un titre ou un autre. Elle pose cependant un certain nombre de problèmes éthiques et méthodologiques. Le premier, et non des moindres, est que la personne enquêtée se retrouve dans une situation où elle se sent 'obligée' de se livrer. Elle risque alors de se sentir gênée, soit par les révélations faites, soit par ses non-dits et ses silences, soit par le simple fait d'avoir été obligée de mentir sur une partie de son vécu. Certains acteurs percevront cet exercice de mémoire de manière moins invasive ; la difficulté pour le chercheur consistera à pouvoir distinguer les expériences individuelles des interprétations imaginées collectivement par la rumeur ou reconstruites à partir d'informations journalistiques ou politiques, par exemple. Un autre dilemme propre aux recherches qui ont pour objet des catégories sociales produites par les intervenants humanitaires (" réfugiés ", "vulnérables", " enfants soldats", " ex-combattants", etc.) est d'arriver à s'extraire de leur approche normative tout en essayant de capitaliser sur les savoirs produits par ces institutions. Comment s'assurer en effet de la qualité des données collectées dans de tels contextes ultramilitarisés, ultra-politisés, ultra-polarisés et souvent sous perfusion humanitaire ? Si l'on considère la place prise par le discours institutionnel dans les zones soumises à l'intervention humanitaire et la présence physique de 
certains de ces acteurs dans des zones jusqu'alors évitées par les chercheurs parce que jugées trop dangereuses, la littérature grise de ces institutions constitue une mine d'informations. Pour s'assurer des données de qualité, il faut d'une part composer avec la multiplicité des acteurs et d'autre part utiliser le dispositif humanitaire en place comme gatekeeper, c'est-à-dire comme un moyen d'accès à un certain nombre d'informateurs par le biais des projets mis en place (santé, réinsertion sociale ou économique de certains groupes, etc.). S'il y a un réel risque de biais méthodologique à cause d'un engagement institutionnel mal distancié, l'entrée par les interventions humanitaires offre néanmoins des opportunités d'accès à un vivier important d'informateurs potentiels, relativement disponibles et ouverts à la discussion.

\section{Peut-on vraiment enquêter sur tout?}

Enfin, à côté des risques liés au contexte immédiat, d'autres risques sont liés à la nature de l'objet de recherche qui peut être politiquement " sensible ", socialement "illégitime " ou religieusement " tabou ». La sensibilité particulière de ces objets de recherche se traduit par des conflits conceptuels qui les rendent hostiles, fuyants, "saturés " d'enjeux sémantiques qui mettent à mal les canons de la méthode (la neutralité du chercheur, la rigueur méthodologique, etc.), comme on le verra dans ce numéro avec les contributions de Ramatou Ouedraogo et de Hadiza Moussa qui ont trouvé leurs objets de recherche pris dans des conflits de significations très tendus. L'un concernait la question de l'avortement dans un contexte de stigmatisation communautaire (Ramatou Ouedraogo) tandis que l'autre concernait le problème de l'excision dans un contexte de religiosité exacerbée (Hadiza Moussa), deux objets d'étude caractérisés par un fort stigma social. L'anthropologie de la santé est une mine en ce domaine. Comment étudier des patients séropositifs, dans des milieux où le SIDA est encore auréolé de croyances populaires ? Comment étudier les interruptions volontaires de grossesse là où l'on restreint juridiquement leur pratique et où les femmes sont exposées à une forte réprobation sociale? Comment arriver à parler des pratiques d'excision dans des contextes où les populations pratiquantes privilégient le repli identitaire et le silence sur le sujet ? 
La contribution de Hadiza Moussa donne un éclairage particulier sur les contraintes subies par le chercheur enquêtant sur les pratiques d'excision au Sahel. Stigmatisé de manière particulièrement négative au Burkina Faso où la pratique est sévèrement réprimée par les autorités locales, cet effet "Burkina » a essaimé dans les pays voisins et notamment au Niger, terrain choisi par l'auteure pour illustrer les précautions méthodologiques nécessaires à l'appréhension d'un tel sujet. La première précaution est de nature sémantique. Partant de son expérience de chercheure-experte associée à un programme éducatif porté par le comité nigérien sur les pratiques traditionnelles (CONIPRAT), l'auteure montre la nécessité d'épurer les questionnaires et les guides d'entretien des termes posant problème (" excision ", " mutilations génitales féminines ", " circoncision »). Une terminologie plus neutre a ainsi été préférée qui privilégie les termes de type " marquage corporel », ce qui permettait d'inclure des pratiques locales répandues telles que les pratiques de scarifications, de piercings et de taillage de dents. La deuxième précaution méthodologique qui a été adoptée a été d'adapter l'approche. Dans un contexte où le chercheur peut être accepté ou rejeté, selon l'étiquette qui lui est associée (si on le perçoit en tant qu'agent répressif par exemple), il était important de former les enquêteurs à aborder le thème de l'excision de manière indirecte dans les lieux d'enquête, en utilisant comme entrée les réflexions personnelles des enquêtés sur les droits humains et en passant complètement sous silence - malgré les dilemmes éthiques que cela impliquait - le fait que l'étude était commissionnée par la CONIPRAT. Sans surprise, les résultats ont été mitigés : certaines personnes pratiquant ou ayant pratiqué l'excision ont accepté de se livrer tandis que d'autres, lassées des critiques toujours plus virulentes de l'UNICEF ou des ONG sur ces pratiques, ont choisi le silence ou le repli sur soi en s'abritant derrière la défense d'une tradition ancestrale revendiquée comme patrimoine culturel.

Dans sa contribution portant sur une expérience d'enquête sur le thème de l'avortement au Burkina Faso, Ramatou Ouedraogo fait part de préoccupations proches de celles de Hadiza Moussa. Comment enquêter sur ce qui peut être considéré comme une douleur pour les uns et une déviance pour les autres ? Comment faire une recherche sur un objet connu de tous mais invisible? Comment investiguer une pratique faisant l'objet d'une "mauvaise foi sociale" ? Sa contribution souligne à quel point le champ de l'avortement au Burkina Faso peut être un lieu de con- 
frontation de convictions diverses où les acteurs naviguent entre non-dits, faux-semblants, censures et suspicions. $\mathrm{Si}$, comme elle le montre, le contexte normatif de l'avortement au Burkina Faso transforme la quête de l'avortement en une sorte de "chemin de croix" fait de souffrance, de solitude, de vulnérabilité, de violence, de danger et de douleurs dans lequel le réseau relationnel joue un rôle important, les jeunes femmes en quête d'avortement tentent généralement leur chance auprès de toutes les personnes susceptibles de les aider dans leur réseau social. Le chercheur se retrouve alors " incorporé " malgré lui dans ce réseau. Dans ses interactions avec ces jeunes femmes burkinabés en quête d'avortement, le chercheur devient alors un recours possible. Elles imaginent qu'il dispose de moyens pour les aider dans leur quête et cherchent à l'impliquer dans leur démarche (en les aidant à trouver un avorteur, en les aidant à prendre leur décision, etc.). Réagir ou non à ces sollicitations, répondre ou non aux attentes des sujets enquêtés peut engendrer une forme de souffrance de la part du chercheur, d'abord au regard de ses propres valeurs, ensuite au regard du risque d'enfreindre une loi s'il se rend complice de certains actes.

Apprendre à gérer les émotions, tant du côté du chercheur que des sujets enquêtés, est un défi permanent tout au long des recherches conduites sur des terrains dangereux ou sur des objets tabous. Dans ce genre de recherches, le chercheur est parfois confronté à des représentations extrêmes de la réalité, à certaines idées et pratiques de la violence. Jusqu'où le chercheur peut-il prétendre suspendre son jugement ? Après combien d'écoutes ? Comment apprendre à canaliser ses émotions dans une méthodologie réflexive ? Plusieurs contributions illustrent ces aspects. Les chercheurs ne sont pas immunisés contre les chocs émotionnels et certains discours restent difficiles à entendre, même pour les esprits les plus ouverts. Wood (2006) est une des premières à reconnaître l'importance des dynamiques émotionnelles; en nier l'impact sur le processus d'analyse peut amener le chercheur à des erreurs de jugement. Dans un article provocateur, Kevin Haggerty (2004), sociologue et criminologue, s'interroge sur la notion de 'harmful' que l'on pourrait traduire dans ce contexte par 'néfaste' ou 'préjudiciable'. Dans l'article, il définit ainsi l'idée d'un projet risqué: 
"Si un projet de recherche pose de plus grands risques que ceux usuels auxquels la personne est confrontée dans sa vie quotidienne, ces risques doivent être atténués par le chercheur; dans le cas contraire, la recherche ne pourra pas être conduite. ${ }^{8}$ (Haggerty, 2004)

Dans les zones devenues inaccessibles pour les chercheurs extérieurs parce que jugées trop dangereuses à certaines périodes en particulier, la question du 'suivi à distance' se pose pleinement.

\section{Jusqu'où peut-on aller sans enquête? Enjeux méthodologiques et éthiques de l'enquête à distance}

Informé d'un contexte "à risque " le chercheur peut donc suspendre son départ en mission de recherche. Dans ce cas, il lui reste la possibilité de s'engager dans une forme "d'enquête à distance" ou de "suivi à distance " de l'enquête. C'est un élément encore très peu exploré à ce jour ; pourtant, cette solution méthodologique est de plus en plus souvent adoptée dans le cas de terrains d'enquêtes devenus 'difficiles' d'accès. L'enquête " à distance " doit être comprise comme la collecte de données par un tiers (généralement un assistant de recherche qui réside in situ, sur le terrain) tandis que le chercheur se trouvant dans l'impossibilité de se rendre personnellement sur le terrain, en raison notamment de I'interdiction de voyager qui est émise par son institution d'appartenance (à cause du caractère jugé " trop dangereux " de la zone), se réserve le travail d'analyse " de loin ". Il s'agit donc d'un dispositif méthodologique qui consiste à s'affranchir de la méthode anthropologique classique pour s'adapter à des contraintes sécuritaires en recherchant des méthodes d'acquisition d'information compatibles avec ses contraintes.

La contribution de Sten Hagberg et Gabriella Körling dans ce numéro est éclairante sur ce point. Leur article montre que l'inaccessibilité temporaire du terrain (le Mali dans leur cas) les a obligés à s'engager dans une démarche méthodologique inattendue, au sens anthropologique du terme,

${ }^{8}$ " In the eventuality that a research project poses a greater risk than what a person might encounter in his/her daily life, these risks must be managed by the researcher or the research cannot be conducted» (traduit par les auteurs). 
mais qui s'est cependant révélée intéressante. Si leur intention de départ était d'explorer l'évolution dans le temps des politiques municipales maliennes, le conflit de 2012 au Mali a empêché la mise en œuvre d'une enquête terrain conventionnelle. Les deux anthropologues ont donc modifié leur prétention initiale et se sont orientés vers une analyse discursive des médias maliens et du discours public. Le changement de démarche a finalement offert des perspectives intéressantes sur la compréhension de la circulation des représentations et des stéréotypes dans les discours locaux. Cette solution méthodologique, pour une discipline qui cultive traditionnellement l'observation longue et directe sur le terrain, propose un positionnement nouveau en réhabilitant, voire en revendiquant, diverses méthodes de 'suivi à distance' et d'analyse discursive comme fondement à l'enquête.

L'autre manière d'envisager le suivi à distance consiste à passer par des tiers pour procéder à la collecte des informations primaires. Sur des terrains difficiles d'accès, cette pratique est assez répandue en anthropologie, comme en témoignent les travaux de nombreux doctorants des pays 'du Sud' qui alimentent les articles de chercheurs 'du Nord'. Ce dispositif pose un certain nombre de questions méthodologiques et éthiques : comment construire un tel partenariat ? Jusqu'où peut aller le chercheur dans la manipulation des données du tiers? Comment valoriser ce travail collectif à sa juste valeur, sans frustrer le travail de collecte ni sous-valoriser l'analyse ? Les pistes de réflexion sont encore vierges sur le sujet. Pour autant, la démarche anthropologique étant basée sur un investissement personnel important en termes de production de données, l'évolution des pratiques de collecte, surtout quand on touche à des terrains dangereux et/ou difficiles d'accès, doit être documentée. La contribution de Sten Hagberg et Gabriella Körling témoigne ainsi d'un courant anthropologique qui explore les implications épistémologiques d'une enquête de terrain au long cours marquée par la présence sporadique de l'ethnographe sur le terrain.

Si la notion d'enquête à distance mérite qu'on s'y attarde, ce n'est pas seulement parce qu'elle permet de résoudre, en partie, certains problèmes d'accès à l'information produite sur le terrain. II y a, selon nous, une autre raison très importante qui est liée aux récentes évolutions des processus et des medias d'interconnexion. En effet, ces dernières années, 
la distance physique entre le "terrain " et "l'université " s'est vue extraordinairement réduite par le formidable développement des moyens électroniques de communication instantanée. Ainsi, Deborah D'AmicoSamuels considère que le " terrain " ne doit plus être réductible aux translations spatiales du chercheur :

"What does 'leave the field' mean in a high-tech, postmodern, and globalized world where even research participants in the remotest areas are accessible by telephone? " (D'Amico-Samuels, 1991 : 83)

S'en tenant à la conception du terrain comme processus ethnographique, elle considère que le terrain est partout :

"There is no division between home and the field because both exist in the same holistic context of globalized power relations. " (ibid.)

Aujourd'hui, des anthropologues de plus en plus nombreux considèrent que, d'un certain point de vue, ils ne quittent plus vraiment le terrain et que, dès lors, leur enquête ne se termine jamais (Watson, $1999: 25)$. Sten Hagberg et Gabriella Körling notent à ce propos qu'un échange téléphonique avec prise de notes, fondé sur des relations construites avec des assistants de recherche lors de missions précédentes sur le terrain, n'est pas si différent d'un entretien réalisé in situ. Pourtant, il y a une distance physique immense entre les interlocuteurs : le chercheur est "chez lui » en Europe et les assistants de recherche sont sur le terrain, " chez eux ", dans un pays en guerre.

\section{Le terrain malgré tout}

Cette nouvelle orientation de l'enquête, paradoxale à maints égards, est lourde de conséquences épistémologiques ainsi qu'en témoignent les travaux récents de Mirjam de Bruijn et Rijk van Dijk (2012). En effet, la caractéristique fondamentale de l'enquête de terrain était bien d'être présent sur le terrain, "being there " (Bradburd, 1988; Wood, 2006), c'est-à-dire sur les lieux de l'enquête. L'enquête à distance oblige donc à se poser la question de la nature des données et des informations ainsi 
recueillies. Entre autres, celle de savoir si la référence à la réalité peut être entièrement contenue dans les mots prononcés à distance ${ }^{9}$ ? S'il s'agit d'informations factuelles et objectivées par le langage, l'entretien à distance, par téléphone ou par recours aux informations produites par d'autres medias, peut être très instructif et productif. Par contre, la présence sur le terrain, in situ, s'avère indispensable à la production de savoirs plus complexes à construire. Ainsi, la découverte de certaines conceptions collectives ou individuelles ne peut se faire autrement que par la pratique d'une interaction quotidienne réfléchie qui permet d'être exposé à des représentations non organisées par des concepts ou des notions précises bien que centrales à la compréhension de la situation étudiée.

De même, seule la présence sur les lieux de l'enquête permet :

" [...] l'apprentissage par la pratique de savoirs faire implicites, incorporés, de connaissances tacites, sous-jacentes à l'exercice d'activités routinières, réalisées par ses interlocuteurs sur le mode de l'évidence, comme allant de soi, et relevant par là d'un certain sens commun partagé, agi au quotidien, et n'étant pas pour cela systématiquement formulé. » (Berger, $2004:$ 80)

L'immersion dans le milieu investigué caractéristique tant de l'observation participante que de l'observation participative et réflexive s'avère irremplaçable car elle fournit des informations sur les non-dits tout en donnant accès à d'autres discours qui corrigent, nuancent, contredisent ou infléchissent celui qui est adressé de manière univoque par téléphone à l'anthropologue ou véhiculé par les medias d'information. La valeur heuristique de l'enquête à distance est donc limitée. Malheureusement, la situation d'enquête de longue durée sur un terrain est aujourd'hui menacée de deux manières. D'une part, à cause des problèmes d'insécurité - que nous venons d'évoquer amplement ici - susceptibles de menacer la vie du chercheur, de ses assistants de recherche ou de ses informateurs et, d'autre part, à cause de la réduction des crédits alloués à l'enquête de terrain qui ont partout tendance à diminuer. Ce contexte politicoéconomique qui pèse sur la recherche et son financement exerce une forte

\footnotetext{
${ }^{9}$ Dans cette situation d'enquête, le chercheur est dans l'incapacité de confronter les discours aux pratiques des acteurs et de valider les informations par une procédure de triangulation simple ou complexe.
} 
contrainte sur l'enquête anthropologique de terrain et oblige de plus en plus les anthropologues à critiquer la méthode ethnographique et à redéfinir et complexifier encore leur dispositif d'enquête sur le terrain. Avec cette question de l'enquête à distance, la revue Anthropologie \& développement ouvre un débat méthodologique qui n'est pas près de se terminer.

\section{Bibliographie}

AGIER M., 1997, " Ni trop près, ni trop loin. De l'implication ethnographique à l'engagement intellectuel », Gradhiva, 21: 69-76.

ALBERA D., 2001, "Terrains minés en ethnologie ", Ethnologie française, 1: 5-13.

BENSA A. et FASSIN E., 2002, "Les sciences sociales face à l'événement ", Terrain, mars, $n^{\circ} 38: 5-20$.

BERGER L., 2004, Les nouvelles ethnologies. Enjeux et perspectives, Paris, Nathan.

BIZEUL D., 2007, "Que faire des expériences d'enquête? Apport et fragilités de I'observation directe ", Revue française de science politique, vol. LVII (1) : 69-83.

BOUMAZA M. et CAMPANA A., 2007, " Enquêter en milieu "difficile". Introduction ", Revue Française de science politique, vol. LVII.

BOUILLON F., FRESIA M. et TALLIO V., 2005, Terrains sensibles : une expérience de l'Anthropologie, Paris, Editions de l'EHESS.

BRADBURD D., 1988, Being There. The Necessity of Fieldwork, Washington, Smithsonian Institution Press.

COPANS J. et GENEST S., 2000, « Présentation. L'anthropologie et le millénaire. Fin de siècle? ", Anthropologie et sociétés, n²4 (1) : 5-14.

D'AMICO-SAMUELS D., 1991, " Undoing Fieldwork: Personal, Political, Theoretical and Methodological Implications ", in F.V. Harrison (ed.), Decolonizing Anthropology: Moving Further Toward an Anthropology for Liberation: 68-87.

DE BRUIJN M.E. et VAN DIJK R., 2012, "Connecting and Change in African Societies: Examples of "Ethnographies of Linking" in Anthropology", Anthropologica, 54. 
HAGERTY K., 2004, " Ethic Creep: Governing Social Science Research in the Name of Ethics », Qualitative Sociology, 27 (4): 391-414.

LE PALEC A. et LUXEREAU A., 1999, " Introduction: ethnographies en situations extrêmes ", Journal des Anthropologues, Situations de violence, $n^{\circ} 76$ : 27-31.

NILAN P., 2002, “'Dangerous Fieldwork' Re-examined: The Question of Researcher Subject Position ", Qualitative Research, 2 (3): 363-386.

NORDSTROM C. et ROBBEN A.C.G.M., 1995, Fieldwork under Fire: Contemporary Studies of Violence and Survival, Berkeley, University of California Press.

PINK S., 2000, " "Informants" Who Come "Home" », in Amit V. (ed.), Constructing the Field: Ethnographic Fieldwork in the Contemporary World, London, Routledge : 96-119.

PINK S. (ed.), 2006, Applications of Anthropology, London, Berghahn Books.

PULMAN B., 1988, "Pour une histoire de la notion de terrain ", Gradhiva, 5 : 21-30.

ROBBEN A.C.G.M. et SLUKA J.A. (eds), 2007, Ethnographic Fieldwork. An Anthropological Reader, Blackwell Publishing, Oxford.

SRIRAM C.L., KING J.C., MERTUS J.A., MARTIN-ORTEGA O. et HERMAN J. (eds), 2009, Surviving Field Research: Working in violent and Difficult Situations, London, Routledge.

WATSON C.W. (ed.), 1999, Being there: fieldwork in anthropology, Coll. Anthropology, culture, and society, London, Sterling, Va., Pluto Press.

WAX R., 1986, Doing Fieldwork, Warning and Advice, Chicago, Chicago University Press.

WEBER F., 2008, "Publier des cas ethnographiques : analyse sociologique, réputation et image de soi des enquêtés ", Genèses, n 70, no $1: 140$ 150.

WOOD E.J., 2006, "The Ethical Challenges of Field Research in Conflict Zones ", Qualitative Sociology, 29 (3): 373-386.

\section{ANANA}

Sylvie Ayimpam est socio-anthropologue à l'Institut des Mondes Africains (IMAf), Aix-Marseille E-mail : ayimpam@mmsh.univ-aix.fr

Magali Chelpi-den Hamer est anthropologue à l'Institut des Mondes Africains (IMAf), Aix-marseille

E-mail : chelpi@mmsh.univ-aix.fr 